\title{
Higher Order Analogues of Tracy-Widom Distributions via the Lax Method
}

\author{
Gernot Akemann and Max R. Atkin \\ Fakultät für Physik, Universität Bielefeld, \\ Postfach 100131, D-33501 Bielefeld, Germany
}

\begin{abstract}
We study the distribution of the largest eigenvalue in Hermitian one-matrix models when the spectral density acquires an extra number of $k-1$ zeros at the edge. The distributions are directly expressed through the norms of orthogonal polynomials on a semi-infinite interval, as an alternative to using Fredholm determinants. They satisfy non-linear recurrence relations which we show form a Lax pair, making contact to the string literature in the early 1990's. The technique of pseudo-differential operators allows us to give compact expressions for the logarithm of the gap probability in terms of the Painlevé XXXIV hierarchy. These are the higher order analogues of the TracyWidom distribution which has $k=1$. Using known Bäcklund transformations we show how to simplify earlier equivalent results that are derived from Fredholm determinant theory, valid for even $k$ in terms of the Painlevé II hierarchy.
\end{abstract}




\section{Introduction}

Random matrices appear in many branches of mathematics, physics and other sciences. Perhaps the best example to illustrate such a breadth of applications is a single formula known as the Tracy-Widom (TW) distribution, and we refer to [1] for an incomplete list. It was first realised as the scaled distribution of the largest eigenvalue of a large complex Hermitian random matrix with Gaussian entries [2] that can be expressed as the derivative of the Fredholm determinant of the Airy-kernel [3]. Apart from its appearance in many other areas this distribution is remarkably robust in the sense that it holds for a much larger class of non-Gaussian distributions, of either invariant [4] or Wigner type [5]; this property is known as universality.

Another striking feature is the relation between the TW distribution and integrable hierarchies of non-linear differential equations, notably the Painlevé hierarchies. A relation between matrix models and integrable hierarchies was first observed in the string literature of the early 1990's where matrix models were applied to two-dimensional quantum gravity, and we refer to [6] for an extensive review. There the possibility of having "multicritical points" was introduced in order to couple matter to gravity. This happens when $k-1$ additional zeros collide with the spectral density - which is the semi-circle in the Gaussian case $(k=1)$. Such scenarios can be realised by fine-tuning non-Gaussian potentials to "critical potentials", see e.g. [7, 8] for a classification and references. In order to have the largest possible set of critical exponents available this also included so-called formal matrix models, where the confining potential is not bounded from below. This allows $k$ to assume any integer value. When considering multicritical models it is usual to study the perturbations of such models away from criticality by suitably scaling some of the parameters in the potential as it approaches its critical form. It should be noted that sometimes in the mathematics literature the term multicritical model is reserved for the case when all possible perturbations are included, however in this article we will use the term multicritical in the sense introduced above; this coincides with the physics literature.

Whereas the limiting kernel of orthogonal polynomials with multicritical potentials has been considered before in mathematics, e.g. in [9, 10], and in the physics literature, see [1] for a comprehensive list of references, it was not until recently that the question about the existence of higher order analogues of TW distributions at such multicritical points was first answered [12]. This also includes a first numerical investigation in [13]. Building on the Riemann-Hilbert analysis of the limiting kernel for $k=3$ [9], in [12 Fredholm determinant theory was used to describe the higher order analogues of TW for any odd $k 1$. They were found to be characterised by a system of equations including the odd members of the Painlevé II hierarchy.

Several alternative derivations of the TW distributions exist, in particular a recent heuristic but very transparent one [14], that avoids the Fredholm determinant formalism. Starting from a Gaussian potential they directly compute the gap probability that the interval $(y, \infty)$ is empty of eigenvalues. It is given in terms of the norms of orthogonal polynomials on the complement $(-\infty, y]$ which satisfy a set of non-linear differential equations. After carefully

\footnotetext{
${ }^{1}$ Note that our convention of counting zeros differs from 12 .
} 
performing the asymptotic analysis of the recursion coefficients in the three-step recurrence relation TW at $k=1$ follows.

Our goal is to generalise the approach [14] to the higher order multicritical cases. However, it rapidly becomes very cumbersome, already when extending their derivation of TW to a generic quartic potential (which only shows its known universality). The main idea in this paper is to cast the recurrence relations into a different set of flow or string equations, that allow to make close contact to integrable hierarchies via the Lax pair. Once these commutation relations become multiplicative we can apply the Lax method to solve them using pseudo-differential operators, following [6, 15]. Ultimately this leads to a hierarchy of differential equations given by the Painlevé XXXIV hierarchy for any integer $k$, in terms of which the logarithm of the gap probability at multicriticality can be expressed. Here we exploit that the limiting case $y \rightarrow \infty$ is well understood in terms of these integrable hierarchies, see e.g. 6]. Furthermore, we manage to prove the equivalence to the subset of odd- $k$ hierarchies that were derived rigorously in [12. The link is provided by a Bäcklund transformation that was explicitly constructed in 16. Applied to our result it leads to all members of Painlevé II including [12]. A shift property of the so-called Lenard differential operators (also known as Gelfand-Dikii polynomials) allows us to simply their result.

The remainder of this paper is organised as follows. In section 2 we review the orthogonal polynomial formalism adopted to [14] and derive our flow and string equation for finite matrix size. The main result is derived in section 3 where the double scaling limit is taken on the string equation, leading to Painlevé hierarchies for the gap probability. Explicit examples including TW are given and the equivalence to [12] is shown, before concluding in section 4. Various technical details are delegated to the appendices.

\section{The Orthogonal Polynomial Formalism}

Consider the following truncated partition function

$$
\begin{aligned}
Z_{N}\left(y ; \alpha,\left\{g_{l}\right\}\right) & \equiv \frac{1}{N !} \int_{-\infty}^{y} \prod_{i=1}^{N} d \lambda_{i} \mathrm{e}^{-N \alpha V\left(\lambda_{i}\right)} \prod_{k>j}^{N}\left(\lambda_{k}-\lambda_{j}\right)^{2} \\
V(\lambda) & \equiv \sum_{l=1}^{\infty} \frac{1}{l} g_{l} \lambda^{l} .
\end{aligned}
$$

Here $V(\lambda)$ is a formal power series and $\alpha>0$ is a real parameter. Both are independent of $y$. When sending the upper integration range $y \rightarrow \infty$ it corresponds to the standard Hermitian one-matrix model $Z_{N}\left(\infty ; \alpha,\left\{g_{l}\right\}\right)$, given in terms of eigenvalues $\lambda_{i}$ of a random matrix $M$. In general we want to compute the probability $\mathbb{P}_{N}\left(\lambda_{\max }<y ; \alpha,\left\{g_{l}\right\}\right)$ that the largest eigenvalue of the random matrix $M$, is less than $y$. It has the following expression,

$$
\mathbb{P}_{N}\left(\lambda_{\max }<y ; \alpha,\left\{g_{l}\right\}\right)=\frac{Z_{N}\left(y ; \alpha,\left\{g_{l}\right\}\right)}{Z_{N}\left(\infty ; \alpha,\left\{g_{l}\right\}\right)},
$$

which is why our definition eq. (2.1) is convenient. One possibility to determine this probability is to introduce orthogonal polynomials for the partition function $Z_{N}\left(\infty ; \alpha,\left\{g_{l}\right\}\right)$ and 
to study their asymptotic behaviour in an appropriate scaling limit. However, we will follow [14] here by introducing polynomials for the truncated partition $Z_{N}\left(y ; \alpha,\left\{g_{l}\right\}\right)$. Formally this could also be formulated in the one-matrix model by adding a hard wall at $y$ to the potential. Introduce a set of polynomials $\left\{\pi_{n}(\lambda): n \in \mathbb{N}\right\}$ such that $\pi_{n}(\lambda)$ is of order $n$ and they are orthonormal with respect to the inner product defined by,

$$
\left\langle\pi_{n} \mid \pi_{m}\right\rangle \equiv \int_{-\infty}^{y} d \lambda \mathrm{e}^{-N \alpha V(\lambda)} \pi_{n}(\lambda) \pi_{m}(\lambda)=\delta_{n m}
$$

Note that the coefficients in the expression for each $\pi_{n}$ depend on $y, \alpha$ and the potential. Furthermore, we define $h_{n}=h_{n}(y)>0$ by the leading coefficients

$$
\pi_{n}(\lambda)=\frac{1}{\sqrt{h_{n}}} \lambda^{n}+O\left(\lambda^{n-1}\right) .
$$

They are positive being the squared norms of the polynomials in monic normalisation. It can be shown using the orthonormality that [17]

$$
Z_{N}\left(y ; \alpha,\left\{g_{l}\right\}\right)=\prod_{i=0}^{N-1} h_{i}=h_{0}^{N} \prod_{i=1}^{N-1} r_{i}^{N-i}
$$

Here we have defined the ratios

$$
r_{n}=\frac{h_{n}}{h_{n-1}} \quad \text { for } \quad n \geq 1
$$

that will determine the distribution of the largest eigenvalue. It is useful to rewrite $Z_{N}\left(y ; \alpha,\left\{g_{l}\right\}\right)$ as,

$$
\log \left[Z_{N}\left(y ; \alpha,\left\{g_{l}\right\}\right)\right]=N \log \left[h_{0}\right]+N \sum_{i=1}^{N-1}\left(1-\frac{i}{N}\right) \log \left[r_{i}\right] .
$$

In the case of a Gaussian potential $g_{l}=2 \delta_{2, l}$, and $y \rightarrow \infty$ everything is explicitly known for finite- $N$ because the $\pi_{n}$ are proportional to Hermite polynomials, with $h_{n}=n ! \sqrt{\pi} / 2^{n}$ and the ratio assuming the simple form $r_{n}=n / 2$ (we have set $N \alpha=1$ for simplicity).

In order to determine the $r_{i}$ in a general setting we first introduce a set of multiplication and differentiation operators on the ring of polynomials $\pi_{n}$ which form a complete set of functions. We begin with the multiplication operator $B$ :

$$
B_{n m} \pi_{m}(\lambda) \equiv \lambda \pi_{n}(\lambda)=\sqrt{r_{n+1}} \pi_{n+1}(\lambda)+s_{n} \pi_{n}(\lambda)+\sqrt{r_{n}} \pi_{n-1}(\lambda)
$$

where we have used summation conventions for the matrix multiplication on the left hand side. In terms of the inner product this reads

$$
B_{n m}=\left\langle\pi_{n} \mid \lambda \pi_{m}\right\rangle=\sqrt{r_{n+1}} \delta_{n+1, m}+s_{n} \delta_{n, m}+\sqrt{r_{n}} \delta_{n-1, m} .
$$

The fact that $B$ is tridiagonal follows from the three step recurrence relation that arbitrary orthogonal polynomials with weights on the real line satisfy; this includes the case of the 
truncated integral in (2.1). Besides $r_{n}$ we encounter here a second recurrence coefficient, $s_{n}=\left\langle\pi_{n} \mid \lambda \pi_{n}\right\rangle$, that must be determined in principle. Obviously $B$ is a symmetric matrix as $B_{n m}=\left\langle\pi_{n} \mid \lambda \pi_{m}\right\rangle=\left\langle\pi_{m} \mid \lambda \pi_{n}\right\rangle$, or in operator language $B=B^{T}$.

Differentiation with respect to the argument of the polynomials is denoted by $A$ :

$$
A_{n m} \pi_{m}(\lambda) \equiv \partial_{\lambda} \pi_{n}(\lambda)
$$

or in terms of the inner product $A_{n m}=\left\langle\pi_{m} \mid \partial_{\lambda} \pi_{n}\right\rangle$. Because the coefficients $h_{n}$ are $\lambda$ independent the polynomial on the right hand side is at most of degree $n-1$, and thus

$$
A_{n, n+k}=0 \quad \text { for } \quad k>-1
$$

or in other words $A$ is strictly lower triangular. In particular for the uppermost non-zero diagonal we have

$$
A_{n, n-1}=\int_{-\infty}^{y} d \lambda \mathrm{e}^{-N \alpha V(\lambda)} \pi_{n-1} \partial_{\lambda}\left(\frac{1}{\sqrt{h_{n}}} \lambda^{n}+O\left(\lambda^{n-1}\right)\right)=n / \sqrt{r_{n}} .
$$

For the Gaussian potential other explicit expressions can be derived. It is easy to see that $A$ and $B$ form canonical commutation relations

$$
[B, A]=1
$$

due to $(B A-A B)_{n m} \pi_{m}=\partial_{\lambda}\left(\lambda \pi_{n}\right)-\lambda \partial_{\lambda} \pi_{n}=\pi_{m} \delta_{n, m}$, upon using the definitions.

Finally we also introduce differentiation with respect to the truncation $y$ denoted by $C$ :

$$
C_{n m} \pi_{m}(\lambda) \equiv \partial_{y} \pi_{n}(\lambda)
$$

or equivalently $C_{n m}=\left\langle\pi_{m} \mid \partial_{y} \pi_{n}\right\rangle$. Now the leading order coefficient containing $h_{n}$ in eq. (2.5) is $y$-dependent, and so the polynomial on the right hand side is at most of degree $n$,

$$
C_{n, n+k}=0 \quad \text { for } \quad k>0 \text {. }
$$

The matrix $C$ is lower triangular including the diagonal, which is given by

$$
C_{n n}=\int_{-\infty}^{y} d \lambda \mathrm{e}^{-N \alpha V(\lambda)} \pi_{n} \partial_{y}\left(\frac{1}{\sqrt{h_{n}}} \lambda^{n}+O\left(\lambda^{n-1}\right)\right)=-\frac{1}{2} \partial_{y} \log \left[h_{n}\right] .
$$

For further explicit expressions for the matrix elements see eq. (2.21) below. Matrix $C$ satisfies the following commutation relations with the multiplication operator $B$

$$
[B, C]=-\partial_{y} B
$$

where we have been careful to remember the $y$-dependence of the recursion coefficients, $(B C-C B)_{n m} \pi_{m}=B_{n l} \partial_{y} \pi_{l}-\partial_{y}\left(\lambda \pi_{n}\right)=B_{n l} \partial_{y} \pi_{l}-\partial_{y}\left(B_{n l} \pi_{l}\right)$. We are now prepared to introduce two composed operators that will play a crucial role in the following, by establishing a link to integrable systems. 


\subsection{The Flow Equation}

We begin with the matrix $P$,

$$
P_{n m} \equiv A_{n m}+C_{n m}-\frac{\alpha N}{2} V^{\prime}(B)_{n m}=\left\langle\pi_{m} \mid\left(\partial_{\lambda}+\partial_{y}-\frac{\alpha N}{2} V^{\prime}(\lambda)\right) \pi_{n}\right\rangle .
$$

It is a simple matter of integration by parts to see that $P$ is anti-symmetric, $P+P^{T}=0$ :

$$
\begin{aligned}
P_{n m}= & \int_{-\infty}^{y} d \lambda \mathrm{e}^{-N \alpha V(\lambda)} \pi_{m}\left(\partial_{\lambda}+\partial_{y}-\frac{\alpha N}{2} V^{\prime}(\lambda)\right) \pi_{n} \\
= & \mathrm{e}^{-N \alpha V(y)} \pi_{m}(y) \pi_{n}(y)-\int_{-\infty}^{y} d \lambda \mathrm{e}^{-N \alpha V(y)}\left(-N \alpha V^{\prime}(\lambda) \pi_{m} \pi_{n}+\pi_{n} \partial_{\lambda} \pi_{m}\right) \\
& +\int_{-\infty}^{y} d \lambda \mathrm{e}^{-N \alpha V(\lambda)}\left(\partial_{y}\left(\pi_{n} \pi_{m}\right)-\pi_{n} \partial_{y} \pi_{m}-\frac{\alpha N}{2} V^{\prime}(\lambda) \pi_{m} \pi_{n}\right) \\
= & -\int_{-\infty}^{y} d \lambda \mathrm{e}^{-N \alpha V(y)} \pi_{n}\left(\partial_{\lambda}+\partial_{y}-\frac{N \alpha}{2} V^{\prime}(\lambda)\right) \pi_{m}=-P_{m n} .
\end{aligned}
$$

Here we have used the fact that,

$$
\begin{aligned}
0 & =\partial_{y} \delta_{n, m}=\partial_{y}\left\langle\pi_{n} \mid \pi_{m}\right\rangle=\mathrm{e}^{-N \alpha V(y)} \pi_{n}(y) \pi_{m}(y)+\int_{-\infty}^{y} d \lambda \mathrm{e}^{-N \alpha V(\lambda)} \partial_{y}\left(\pi_{n} \pi_{m}\right) \\
\Leftrightarrow 0 & =\mathrm{e}^{-N \alpha V(y)} \pi_{n}(y) \pi_{m}(y)+C_{m n}+C_{n m}
\end{aligned}
$$

which we have also expressed in terms of the matrix elements of $C$. Secondly, using (2.12) and (2.16) we have that $P_{n, n+k}=-\frac{N \alpha}{2} V^{\prime}(B)_{n, n+k}$ for all $k>0$, and hence using the antisymmetry of $P$ we find that

$$
P=-\frac{N \alpha}{2}\left(V^{\prime}(B)_{+}-V^{\prime}(B)_{-}\right)
$$

where + and - denote the upper and lower triangular parts of a matrix respectively. Obviously its diagonal part vanishes, and its antisymmetry (given the symmetry of $B$ ) is now manifest. Because we have expressed $P$ only in terms of powers of $B$, which is tridiagonal, we see $P$ has only a finite number of non-zero off diagonals. For the Gaussian potential with $g_{l}=2 \delta_{2, l}$ (that is $\left.V(\lambda)=\lambda^{2}\right)$ for example it reads

$$
\left.P_{n m}\right|_{\text {Gauss }}=-N \alpha\left(\sqrt{r_{n+1}} \delta_{n+1, m}-\sqrt{r_{n}} \delta_{n-1, m}\right) .
$$

Finally, given the commutation relations (2.14) and (2.18) we arrive at

$$
[P, y-B]=\partial_{y}(y-B)=1-\partial_{y} B
$$

This is our first main result of this section, and we will refer to it as the flow equation. Note that this equation implies that the matrices $y-B$ and $P$ form a Lax pair 2 . where

${ }^{2}$ After changing to light cone coordinates $x_{ \pm}=(y \pm \lambda) / 2$ and defining $\hat{B}=y-B$ the eqs. (2.9), (2.19) and (2.24) can be written as standard Lax eqs. $\hat{B} \psi_{n}=2 x_{-} \psi_{n}, P \psi_{n}=\partial_{+} \psi_{n}$ and $\partial_{+} \hat{B}=[P, \hat{B}]$ where $\psi_{n}=\pi_{n} \exp [-N \alpha V / 2]$. 
we have added a trivial zero, $0=[P, y]$, to bring it to that form. The flow equation (2.24) provides explicit relations among the recurrence coefficients $r_{n}$ and $s_{n}$, including derivatives with respect to $y$. To give an example we again consider the Gaussian case. Inserting (2.10) and $P$ in the form of (2.23) into (2.24) we obtain five equations. Those for $m=n \pm 2$ are identically satisfied, the equations for $m=n \pm 1$ are the same, and thus we are left with the following two equations for indices $m=n-1$ and $m=n$ :

$$
\begin{aligned}
s_{n}-s_{n-1} & =-\frac{1}{2 \alpha N} \partial_{y} \log \left[r_{n}\right], \\
r_{n+1}-r_{n} & =\frac{1}{2 \alpha N}\left(1-\partial_{y} s_{n}\right) .
\end{aligned}
$$

The first of this set of equations already appeared in [14], however the second is new. The equivalence of our flow equations to [14] is shown in Appendix A. The advantage of our approach is that we may obtain such recursion relations for any potential. Furthermore, we can see that our recursion relations enjoy an explicit link to integrable systems defined by the Lax pair $y-B$ and $P$. Indeed we can see this connection in this particular example as it is trivial to eliminate $s_{n}$, thereby obtaining,

$$
r_{n+1}+r_{n-1}-2 r_{n}=\frac{1}{4 \alpha^{2} N^{2}} \partial_{y}^{2} \log \left[r_{n}\right] .
$$

By substituting $r_{n}=\exp \left[\phi_{n}\right]$ we see that this is equivalent to the difference of the Toda lattice equation at two neighbouring lattice sites.

\subsection{The String Equation}

The second operator we introduce is the matrix $H$. It will lead to a so-called string equation that is algebraic in $r_{n}$ and $s_{n}$. It is defined as,

$$
\begin{aligned}
H_{n m} & \equiv(A(B-y))_{n m}-\frac{N \alpha}{2}\left(V^{\prime}(B)(B-y)\right)_{n m}+\frac{1}{2} \delta_{n m} \\
& =\int_{-\infty}^{y} d \lambda \mathrm{e}^{-N \alpha V(\lambda)} \pi_{m}\left((\lambda-y)\left(\partial_{\lambda}-\frac{\alpha N}{2} V^{\prime}(\lambda)\right)+\frac{1}{2}\right) \pi_{n} .
\end{aligned}
$$

It is easy to see that $H$ is antisymmetric, $H+H^{T}=0$, by applying the following identity in terms of a total derivative:

$$
\begin{aligned}
0 & =\int_{-\infty}^{y} d \lambda \partial_{\lambda}\left[(\lambda-y) \mathrm{e}^{-N \alpha V(\lambda)} \pi_{n}(\lambda) \pi_{m}(\lambda)\right] \\
& =\int_{-\infty}^{y} d \lambda \mathrm{e}^{-N \alpha V(\lambda)}\left[\pi_{n} \pi_{m}+(\lambda-y)\left(-N \alpha V^{\prime}(\lambda) \pi_{n} \pi_{m}+\left(\partial_{\lambda} \pi_{n}\right) \pi_{m}+\pi_{n}\left(\partial_{\lambda} \pi_{m}\right)\right)\right] \\
& =H_{n m}+H_{m n} .
\end{aligned}
$$

Because $A$ is strictly lower triangular and $B$ tridiagonal we have that its product does not

contribute to the + part of $H$, hence $H_{n, n+k}=-\frac{N \alpha}{2}\left(V^{\prime}(B)(B-y)\right)_{n, n+k}$ for all $k>0$. Due to the antisymmetry of $H$ we thus arrive at

$$
H=-\frac{N \alpha}{2}\left(\left(V^{\prime}(B)(B-y)\right)_{+}-\left(V^{\prime}(B)(B-y)\right)_{-}\right) \text {. }
$$


This shows that also $H$ only has a finite number of non-zero off-diagonals, being a multiple of $B$ in its + and - part. For the Gaussian potential it reads

$$
\begin{aligned}
\left.H_{n m}\right|_{\text {Gauss }}= & -N \alpha\left(\sqrt{r_{n+2} r_{n+1}} \delta_{n+2, m}+\sqrt{r_{n+1}}\left(s_{n+1}+s_{n}-y\right) \delta_{n+1, m}\right) \\
& +N \alpha\left(\sqrt{r_{n}}\left(s_{n}+s_{n-1}-y\right) \delta_{n-1, m}+\sqrt{r_{n} r_{n-1}} \delta_{n-2, m}\right) .
\end{aligned}
$$

Furthermore, from the definition (2.28) and the commutation relations (2.14) and (2.18) we obtain

$$
[B-y, H]=B-y,
$$

which is the second result of this section (where we have added a trivial zero, $0=[-y, H]$ ). We shall refer to it as the string equation and it provides relations among the recurrence coefficients, for any potential $V$, which are algebraic and no longer contain derivatives. Let us give again the Gaussian example. Plugging eq. (2.10) and (2.31) into the string equation we obtain five equations, two of which for $m=n \pm 2$ are identically satisfied. The ones for $m=n \pm 1$ are identical and we are left with the two equations for $m=n+1$ and $n=m$ which read,

$$
\begin{aligned}
s_{n+1}^{2}-s_{n}^{2}+r_{n+2}-r_{n}-y\left(s_{n+1}-s_{n}\right) & =\frac{1}{N \alpha}, \\
r_{n+1}\left(s_{n+1}+s_{n}-y\right)-r_{n}\left(s_{n}+s_{n-1}-y\right) & =\frac{1}{2 N \alpha}\left(s_{n}-y\right) .
\end{aligned}
$$

Finally we note that the two operators $P$ and $H$ are related. Because multiplication of matrices and taking their upper $(+)$ or lower $(-)$ part in general do not commute we derive the following relation in Appendix $\mathrm{B}$;

$$
\begin{aligned}
H & =(B-y) P-(B P)_{\mathrm{d}}-\left(B_{+}-B_{-}\right) \frac{N \alpha}{2} V^{\prime}(B)_{\mathrm{d}} \\
& =\frac{1}{2}\{B, P\}-y P-\frac{N \alpha}{4}\left\{B_{+}-B_{-}, V^{\prime}(B)_{\mathrm{d}}\right\},
\end{aligned}
$$

where the subscript $d$ denotes the diagonal part of a matrix. In the second line we have made the anti-symmetry of $H$ manifest, by decomposing it into its (vanishing) symmetric part and its anti-symmetric part. This relation will be important when taking the double scaling limit in the next section.

\section{Double Scaling Limit and the Lax Method}

In this section we will take the large- $N$ limit and determine the asymptotic recurrence coefficients. This will give the distribution of the largest eigenvalue by differentiating (2.3). In the case of a Gaussian potential and $y \rightarrow \infty$ it is well known that the limiting spectral density $\rho(\lambda)$ is given by the Wigner semi-circle that vanishes as a square root at its end points. By taking the large- $N$ limit while simultaneously zooming into the vicinity of the right end point and letting $y$ approach the same end point - hence the name double scaling 
limit (d.s.l) - the distribution of the largest eigenvalue can be found. In [14] this was done using in an approach using the truncated partition function (2.1) which we generalise here.

For non-Gaussian potentials it is well known [7, 8, how to construct multicritical potentials $V_{k, k^{\prime}}$, such that the spectral density behaves as $\rho(\lambda) \sim\left(2 a_{+}-\lambda\right)^{k-1 / 2}\left(\lambda-2 a_{-}\right)^{k^{\prime}-1 / 2}$. Here $\left(2 a_{-}, 2 a_{+}\right)$is the support of $\rho$ which we will assume to be of single interval or one-arc type in all the following, and the integers $k, k^{\prime}=1,2, \ldots$ label the degree of multicriticality, following ref. [8]. So for the Gaussian potential we have $\left(k, k^{\prime}\right)=(1,1)$ with $a_{+}=a_{-}=1$ 3. For these critical points a non-trivial d.s.l can be taken if we make the following scaling ansatz [18],

$$
\begin{aligned}
\xi & =\xi_{c}-a^{k} x \\
N & =a^{-(2 k+1) / 2}
\end{aligned}
$$

where $a$ is the scaling variable given in fractional powers of $N$ and $n / N \rightarrow \xi$ becomes a continuous variable 4 . In addition we make the following general ansatz for our recurrence coefficients $r_{n}$ and $s_{n}$ :

$$
\begin{aligned}
& r(\xi, y)=r_{c}\left(1+a \rho_{1}(x, s)+O\left(a^{3 / 2}\right)\right), \\
& s(\xi, y)=\sqrt{r_{c}}\left(s_{c}+a \sigma_{1}(x, s)+O\left(a^{3 / 2}\right)\right) .
\end{aligned}
$$

It will also be useful to introduce the functions,

$$
u_{1} \equiv \rho_{1}+\sigma_{1}, \quad v_{1} \equiv \rho_{1}-\sigma_{1}
$$

In [4] it was shown that for a one-arc support and analytic potentials the $r_{n}$ and $s_{n}$ indeed become single valued functions. We assume in the following that this remains true for a one-arc support with finite $y$. The d.s.l in the case $y=\infty$ is fully reviewed in Appendix C. However, to quickly recap here; by substituting the above scaling ansatz into the $y=\infty$ string equations found in Appendix $\mathbb{C}$, or alternatively using pseudo-differential operators to solve the same string equation, one finds the result assuming $k^{\prime}<k$,

$$
\begin{aligned}
\mathcal{L}_{k}\left[u_{1}(x, \infty)\right] & =x / 2, \\
\mathcal{L}_{k^{\prime}}\left[v_{1}(x, \infty)\right] & =0
\end{aligned}
$$

The $\mathcal{L}_{k}$ are the Lenard differential operators introduced in the next subsection, see (3.16). In the remainder of this section we argue that the scaling ansatz given in (3.2) together with (3.1), augmented to,

$$
\begin{aligned}
\xi & =\xi_{c}-a^{k} x \\
y & =y_{c}+a c_{1} s \\
N & =a^{-(2 k+1) / 2}
\end{aligned}
$$

where $\xi_{c}=1, c_{1}$ is an arbitrary constant to be fixed later, and $s$ is the microscopic variable that describes the distribution of the largest eigenvalue around the right endpoint of the

\footnotetext{
${ }^{3}$ Note that in [12] the Gaussian case is labelled by $l=k-1=0$, and that only critical points with our $k$ taking odd values are considered.

${ }^{4}$ Note that we always have $\xi_{c}=1$. This will be justified later.
} 
support $y_{c}\left(=2 a_{+}\right)$, gives a non-trivial scaling limit in the case of finite $y$. Furthermore for general $k^{\prime}<k$ we derive the equation satisfied by $u_{1}$ and argue that the equation (3.5) is still valid. Each of these results are also derived in Appendix D in a more rigorous manner.

For a particular potential $V_{k, k^{\prime}}$ we could of course follow [14] and insert (3.6) and (3.2) into either the flow or string equation (2.24) or (2.32) and then Taylor expand in order to derive the relevant differential equations for the scaling functions $u_{i}$ and $v_{i}$. However for general $k$ we require a general method that can uncover the structure of such a hierarchy of equations. The particular approach we adopt here is to follow the method of Appendix C and solve (2.32) using pseudo-differential operators. For a review of this technique we refer to [6] as well as to [15] for its relation to the Lax method.

\subsection{Scaled String Equation and Lenard Differential Operators}

In the large- $N$ d.s.l our matrices $B, P$ and $H$ which all have only a finite number non-zero off diagonals will scale to differential operators of finite order. The commutation relations that we derived in the previous section will then allow us to directly obtain a differential equation for the scaling function. First consider the scaling limit of the matrix $B$. Using our general scaling ansatz (3.6) it scales for all $k$ to [6],

$$
B_{n m} \pi_{m} \rightarrow\left(B_{c}+a \sqrt{r_{c}}\left(d^{2}+u_{1}(x)\right)+O\left(a^{2}\right)\right) \pi_{n}
$$

where $B_{c}=\sqrt{r_{c}}\left(2+s_{c}\right)$ is a constant operator and we have defined $d \equiv d / d x$. For later convenience we also define

$$
\mathcal{B} \equiv d^{2}+u_{1}(x)
$$

The fact that these two terms contributing to $\mathcal{B}$ are of the same order fixes the scaling relation in (3.6) in $\xi$ and $N$ as functions of $a$.

For a given potential the matrix $H$ will scale to a differential operator of a fixed degree, say $m$. Assuming that $H$ has a definite scaling dimension it will be fully determined by the degree $m$,

$$
H \rightarrow a^{(m-2 k-1) / 2} c_{1} c_{2}\left(d^{m}+\ldots\right)+O\left(a^{(m-2 k-1) / 2+1}\right) \equiv a^{(m-2 k-1) / 2} \mathcal{H}+O\left(a^{(m-2 k-1) / 2+1}\right),(3
$$

where $c_{2}$ is a constant. Here we have used the result from the previous calculation that every power of $d$ comes with a scaling dimension $a^{1 / 2}$, and we have multiplied by the extra power of $N$ in front of the potential in (2.30). We may therefore write (2.32) $[B, H]=B-y$ as follows,

$$
a\left(a^{(m-2 k-1) / 2}[\mathcal{B}, \mathcal{H}]+O\left(a^{(m-2 k-1) / 2+1}\right)\right)=a\left(d^{2}+u_{1}-s\right)+O\left(a^{2}\right),
$$

where we have dropped the trivial constant part $B_{c}-y_{c}$ that has to be satisfied. Here we have fixed $c_{1}=\sqrt{r_{c}}$ for convenience. We see that in order to get a non-trivial scaling limit we require $m=2 k+1$. To go further let

$$
\mathcal{H} \equiv \overline{\mathcal{H}}+\frac{1}{4}\{x, d\}
$$


where $\{$,$\} denotes the anti-commutator. A short calculation shows that$

$$
[\mathcal{B}, \overline{\mathcal{H}}]=\frac{1}{2} x u_{1}^{\prime}(x)+u_{1}(x)-s .
$$

The right hand side of (3.12) is now a multiplicative operator and we can therefore directly apply the technique of pseudo-differential operators reviewed in [6] to find an expression for $\overline{\mathcal{H}}$ such that the left hand side of (3.12) is also a multiplicative operator. Indeed it is known that in general,

$$
\overline{\mathcal{H}}=\sum_{n=1}^{k+1} b_{n}\left(\mathcal{B}^{(2 n-1) / 2}\right)_{+},
$$

where here the subscript + denotes the local part of the operator $\mathcal{B}^{n-1 / 2}$, which contains up to order $2 n-1$ differential operators, $d^{2 n-1}$. The terms in (3.13) for which $n<k+1$ will have a scaling dimension incompatible with $\overline{\mathcal{H}}$ unless the coefficients $b_{n}$ also come with a scaling dimension. The only parameter with a scaling dimension in the expression (2.35) is $y$ and therefore the most general solution for $\overline{\mathcal{H}}$ is,

$$
\overline{\mathcal{H}}=c_{1} c_{2}\left(\left(\mathcal{B}^{(2 k+1) / 2}\right)_{+}-b_{k} c_{2}^{-1} s\left(\mathcal{B}^{(2 k-1) / 2}\right)_{+}\right) .
$$

This form which we have deduced here on the ground of general scaling arguments is derived in Appendix $\mathrm{D}$ based on the relation (2.35) between $H$ and $P$ from the previous section. There it is also shown that the relative coefficient, $b_{k} c_{2}^{-1}$, is unity. Finally we can use the known fact that [6]

$$
\left[\mathcal{B},\left(\mathcal{B}^{(2 l-1) / 2}\right)_{+}\right]=-4^{1-l} \mathcal{L}_{l}^{\prime}\left[u_{1}\right]
$$

where $\mathcal{L}_{l}$ is the Lenard differential operator and $\mathcal{L}_{l}^{\prime}$ denotes its $d$-derivative. The Lenard differential operator is related to the Gelfand-Dikii polynomials $R_{l}$ by a trivial rescaling, $\mathcal{L}_{l}=4^{l} R_{l}$. It is defined by the following recursion relation 5 ,

$$
\mathcal{L}_{l+1}^{\prime}[f]=d \mathcal{L}_{l+1}[f]=\left(d^{3}+4 f d+2 f^{\prime}\right) \mathcal{L}_{l}[f] \quad \text { and } \quad \mathcal{L}_{0}=\frac{1}{2} .
$$

Because this only determines $\mathcal{L}_{l}^{\prime}$ one also requires the condition that $\mathcal{L}_{l}[0]=0$ for all $l$. We may thus write (3.12) as

$$
-4^{-k} c_{1} c_{2}\left(\mathcal{L}_{k+1}^{\prime}\left[u_{1}\right]-4 s \mathcal{L}_{k}^{\prime}\left[u_{1}\right]\right)=\frac{1}{2} x u_{1}^{\prime}(x)+u_{1}(x)-s .
$$

We now make use of the fact that under a rescaling of the variables, $x \rightarrow \rho^{-1} x, u_{1} \rightarrow \rho^{2} u_{1}$ and $s \rightarrow \rho^{2} s$, we have $\mathcal{L}_{l}\left[u_{1}\right] \rightarrow \rho^{2 l} \mathcal{L}_{l}\left[u_{1}\right]$. Setting 6 ,

$$
\rho=-2^{\frac{2 k-1}{2 k+1}}\left(c_{1} c_{2}\right)^{\frac{-1}{2 k+1}},
$$

\footnotetext{
${ }^{5}$ Note that in the notation of [6] our $u_{1}(x) \rightarrow-u(x)$, with $\mathcal{B}=d^{2}+u_{1} \rightarrow Q=d^{2}-u$.

${ }^{6}$ Note here the minus sign is necessary to cancel the minus sign on the RHS of the preceeding equation. This extra minus will later appear in the final expression for the distribution by changing the integration region from $[0, \infty)$ to $(-\infty, 0]$
} 
we obtain the equation,

$$
\mathcal{L}_{k+1}^{\prime}\left[u_{1}\right]-4 s \mathcal{L}_{k}^{\prime}\left[u_{1}\right]=x u_{1}^{\prime}(x)+2 u_{1}(x)-2 s .
$$

This is the differential equation our scaling function has to satisfy for the $k$ th multicritical point. From [16] we see it to be a modification of the Painlevé XXXIV hierarchy. For the first few values of $k$ it takes the form,

$$
\begin{array}{ll}
k=1: & u_{1}^{(3)}+6 u_{1} u_{1}^{\prime}-4 s u_{1}^{\prime}=x u_{1}^{\prime}(x)+2 u_{1}(x)-2 s, \\
k=2: & u_{1}^{(5)}+10\left(u_{1}^{\prime}\left(3 u_{1}^{2}+2 u_{1}^{\prime \prime}\right)+u_{1} u_{1}^{(3)}\right)-4 s\left(u_{1}^{(3)}+6 u_{1} u_{1}^{\prime}\right)=x u_{1}^{\prime}(x)+2 u_{1}(x)-2 s, \\
k=3: & u_{1}^{(7)}+70 u_{1}^{\prime \prime} u_{1}^{(3)}+42 u_{1}^{\prime} u_{1}^{(4)}+14 u_{1} u_{1}^{(5)}+70\left(\left(u_{1}^{\prime}\right)^{3}+4 u_{1} u_{1}^{\prime} u_{1}^{\prime \prime}+u_{1}^{2} u_{1}^{(3)}\right)+140 u_{1}^{3} u_{1}^{\prime} \\
& -4 s\left(u_{1}^{(5)}+10\left(u_{1}^{\prime}\left(3 u_{1}^{2}+2 u_{1}^{\prime \prime}\right)+u_{1} u_{1}^{(3)}\right)\right)=x u_{1}^{\prime}(x)+2 u_{1}(x)-2 s,
\end{array}
$$

where we emphasise that $s$ plays the role of a parameter and all differentials are with respect to $x$. Furthermore, from (2.30) we may read off the d.s.l of $P$. We may write (2.30) as,

$$
H=-\frac{N \alpha}{2}\left(\left(V^{\prime}(B) B\right)_{+}-\left(V^{\prime}(B) B\right)_{-}\right)-y P
$$

so $y$ only appears in the term $-y P$. If we assume $P$ scales as $P \rightarrow a^{-\Delta} \mathcal{P}$, where $\Delta$ is its scaling dimension we see that,

$$
-y P \rightarrow-\left(y_{c}+c_{1} s a\right) a^{-\Delta} \mathcal{P}
$$

In order for the term containing $s$ to match the term appearing in (3.14) we conclude $\Delta=1$ and $\mathcal{P}=c_{2}\left(\mathcal{B}^{(2 k-1) / 2}\right)_{+}$. Note that the leading order term which scales as $a^{-\Delta}$ must cancel against the other terms in (3.23). If we now substitute the d.s.l of $P$ together with the usual scaling ansatz into the flow equation (2.24) and use (3.15) we obtain,

$$
\partial_{s} u_{1}(x, s)=\partial_{x}\left(x-2 \mathcal{L}_{k}\left[u_{1}\right]\right) \text {. }
$$

This equation will be useful in the next subsection in order to prove equivalence with the results of [12]. This equation together with (3.19) are the main results of this subsection.

\subsection{Higher Order Analogues of Tracy Widom and Examples}

Now we consider the d.s.l of the expression (2.8). First we take $N \rightarrow \infty$ to obtain,

$$
\log \left[Z_{N}\left(y ; \alpha,\left\{g_{l}\right\}\right)\right]=N^{2} \int_{0}^{1} d \xi(1-\xi) \log [r(\xi, y)]+O(1 / N) .
$$

Note that this formula justifies setting $\xi_{c}=1$; any non-analyticity of $r(\xi, y)$ will only affect the partition function if it occurs in the range $[0,1]$, hence as we approach the critical point 
we must zoom in on the end point of the integration region [6]. If we now substitute the scaling ansatz for general $k$, the above takes the form,

$$
\log Z^{(k)}(s)=-\int_{-\infty}^{0} d x \frac{x}{2}\left(u_{1}(x, s)+v_{1}(x, s)\right),
$$

where we have introduced the scaled partition function $\log Z^{(k)}(s)$ for the $k$ th multicritical point and the limits have acquired an extra minus sign due to the rescaling (3.18). In (3.19) we have only an equation for $u_{1}(x, s)$ and so must specify $v_{1}(x, s)$. In the Appendix we show that the $v_{1}$ in fact satisfies (3.5) for finite $y$. This behaviour is physically resonable as this equation depends only on the behaviour of the eigenvalue density at the end of the support at which the infinite wall is not imposed. Since the modification due to $y$ being finite only affects the local behaviour of the eigenvalue density at the other end of the support this will not have an effect at the opposite end and therefore we expect (3.5) still holds. Imposing the same boundary conditions on $v_{1}$ as the $y \rightarrow \infty$ case, we have for the double scaling limit of the gap probability,

$$
\log \mathbb{P}^{(k)}(s)=-\int_{-\infty}^{0} d x \frac{x}{2}\left(u_{1}(x, s)-u_{1}(x, \infty)\right),
$$

where $u_{1}(x, \infty)$ is the special function appearing in the expression (3.4) i.e. it solves the $k$ th member of the Painlevé I hierarchy. The expression (3.28) together with (3.19) constitute the main result of this paper; they give an expression for the $k$ th multicritical gap probability and hence higher order analogues of the TW distribution. Indeed we can give a useful expression for the distribution of the largest eigenvalue using (3.28), we have,

$$
\frac{d}{d s} \log \mathbb{P}^{(k)}(s)=-\int_{-\infty}^{0} d x \frac{x}{2} \partial_{s} u_{1}(x, s) .
$$

Using (3.25) in (3.29) we have,

$$
\frac{d}{d s} \log \mathbb{P}^{(k)}(s)=-\int_{-\infty}^{0} d x\left(\mathcal{L}_{k}\left[u_{1}\right]-\frac{1}{2} x\right) .
$$

where we have integrated by parts assuming that the boundary conditions on $u_{1}$ cause the boundary terms to vanish. The requirement that the boundary terms vanish implies that as $x \rightarrow-\infty$ we have $u_{1}(x, s) \rightarrow u_{1}(x, \infty)+\delta u_{1}(x, s)$, where $\delta u_{1}(x, s)$ is a subleading contribution containing the $s$ dependence. We note here that this boundary condition is compatible with (3.19) since $u_{1}(x, \infty)$ is in fact a solution to (3.19). We do not discuss the boundary condition for $\delta u_{1}(x, s)$ here, instead we appeal to [12] in which a proposal was made for the appropriate asymptotic behaviour of the solution. In the next subsection we relate our results to theirs; one could then in principle convert their boundary conditions into a condition on $\delta u_{1}(x, s)$.

As an example we now reproduce the standard Tracy-Widom law. For $k=1$ we have that,

$$
\mathcal{L}_{1}\left[u_{1}(x, \infty)\right]=\frac{x}{2} \Rightarrow u_{1}(x, \infty)=\frac{x}{2}
$$


and $u_{1}(x, s)$ satisfies (3.19) for $k=1$ which can be written as,

$$
u_{1}^{(3)}+6 u_{1} u_{1}^{\prime}-(x+4 s) u_{1}^{\prime}-2 u_{1}+2 s=0,
$$

where all differentials are with respect to $x$. Although the choice of normalisation for $u_{1}$, $x$ and $s$ in the main text gives the cleanest results for general $k$, it differs from the choice made by Tracy and Widom [2]. We therefore perform some rescaling, in particular let $u_{1}(x, s)=\gamma^{2} \bar{u}\left(\gamma x, \gamma^{-2} s\right)$ with $\gamma=-2^{-1 / 3}$. Then (3.32) becomes,

$$
\bar{u}^{(3)}(x, s)+2 \bar{u}(x, s)\left(2+3 \bar{u}^{\prime}(x, s)\right)+2(x-2 s) \bar{u}^{\prime}(x, s)-4 s=0 .
$$

Finally, following [14] let $-2 q(x, s)^{2}=\bar{u}(x, s)+x$, substituting this into the above we find firstly that $q(x, s)=q(x+s, 0) \equiv q(x+s)$ and secondly it may be written in the form,

$$
q(x) W^{\prime}(x)=-3 q^{\prime}(x) W(x),
$$

where $W(x) \equiv q^{\prime \prime}(x)-2 q(x)^{3}-x q(x)$. This then can be integrated to show that $q(x)$ satisfies Painlevé II with $\alpha=0$. We now may write (3.28) as,

$$
\log \mathbb{P}^{(k)}(s)=-\int_{-\infty}^{0} d x \frac{x}{2}\left(u_{1}(x, s)-\frac{x}{2}\right)=-\int_{-\infty}^{0} d x \frac{x}{2}\left(\gamma^{2} \bar{u}\left(\gamma x, \gamma^{-2} s\right)-\frac{x}{2}\right) .
$$

Making the change of variables $\bar{x}=\gamma x$ we find,

$$
\begin{aligned}
\log \mathbb{P}^{(k)}(s) & =\int_{0}^{\infty} d \bar{x} \frac{\bar{x}}{2}\left(\bar{u}\left(\bar{x}, \gamma^{-2} s\right)+\bar{x}\right)=-\int_{0}^{\infty} d x x q\left(x+\gamma^{-2} s\right)^{2}, \\
\Rightarrow \log \mathbb{P}^{(k)}\left(\gamma^{2} s\right) & =\int_{s}^{\infty} d x(s-x) q(x)^{2},
\end{aligned}
$$

which coincides with the TW distribution.

\subsection{Relation to Painlevé II via Bäcklund Transformations}

In this section we relate our string equation (3.19) to the Painlevé II hierarchy, thereby making contact with the results of [12]. We proceed using the Bäcklund transformations introduced in [16] which we now review.

We consider two Bäcklund transformations defined by the equations,

$$
2 \mathcal{L}_{k}\left[u_{1}\right]-x=2 \psi(x)^{2} \quad \text { and } \quad \psi^{\prime \prime}(x)+\left(u_{1}-s\right) \psi(x)=0
$$

and

$$
2 \mathcal{L}_{k}\left[W^{\prime}-W^{2}+s\right]-x=2 \psi(x)^{2} \quad \text { and } \quad \psi^{\prime}(x)+W \psi(x)=0 .
$$

In the above equations we have introduced an auxilary variable $\psi$ which will prove convient for comparison with [12]. However, ultimately we are interested in the composition of the 
above Bäcklund transformations in order to obtain a relation between the quantity $u_{1}$ and the new variable $W$.

We now consider the relation between $u_{1}$ and $\psi$ generated by (3.37), by eliminating $u_{1}$ and $\psi$ respectively, as done in [16]. Eliminating $\psi$ in (3.37) yields,

$$
K_{n} K_{n}^{\prime \prime}-\frac{1}{2}\left(K_{n}^{\prime}\right)^{2}+2\left(u_{1}-s\right) K_{n}^{2}=0
$$

where we have introduced the useful quantity $K_{n}\left[u_{1}\right]=2 \mathcal{L}_{k}\left[u_{1}\right]-x$. Differentiating this we obtain,

$$
\left(d^{3}+4 u_{1} d+2 u_{1}\right) \mathcal{L}_{k}\left[u_{1}\right]-4 s \mathcal{L}_{k}^{\prime}\left[u_{1}\right]=x u_{1}^{\prime}+2 u_{1}-2 s,
$$

which using the Lenard recursion formula gives (3.19). On the other hand eliminating $u_{1}$ gives,

$$
2 \mathcal{L}_{k}\left[s-\frac{\psi^{\prime \prime}}{\psi}\right]-x=2 \psi^{2}(x)
$$

The solutions of (3.40) and (3.41) are therefore related by the Bäcklund transformation (3.37).

We now perform the same calculation using (3.38). Eliminating $W$ yields again (3.41), whereas eliminating $\psi$ gives,

$$
(d+2 W) \mathcal{L}_{k}\left[W^{\prime}-W^{2}+s\right]=x W+\frac{1}{2} .
$$

The solutions of (3.41) and (3.42), and hence the solutions of (3.40) and (3.42), are therefore related by the Bäcklund transformation (3.37) and (3.38). The equation (3.42) is closely related to the Painlevé II equation with $\alpha=-1 / 2$. We can make this connection more explicit by using the lemma,

$$
\mathcal{L}_{k}[u(x)+z]=\sum_{j=0}^{k}(4 z)^{k-j} \frac{\Gamma(k+1 / 2)}{\Gamma(k-j+1) \Gamma(j+1 / 2)} \mathcal{L}_{j}[u(x)],
$$

where $z$ is a constant. This lemma can be verified by induction as it is shown in Appendix E using the Lenard recursion relation and seems not to have appeared in the literature before. Hence (3.42) can be written,

$$
(d+2 W) \mathcal{L}_{k}\left[W^{\prime}-W^{2}\right]+\sum_{j=0}^{k-1} \tau_{j}\left(2^{(4 k-2) /(2 k+1)} s\right)(d+2 W) \mathcal{L}_{j}\left[W^{\prime}-W^{2}\right]=x W+\frac{1}{2}
$$

where

$$
\tau_{j}(s)=(2 j+1) 2^{(2 k-4 j-1) /(2 k+1)} \frac{\Gamma(k+1 / 2)}{\Gamma(k-j+1) \Gamma(j+3 / 2)} s^{k-j}
$$


corresponds to the $\tau_{j}$ in [12] if $k$ is odd, and the set of parameters $t_{l}$ there is set to zero. In order to study the distribution of the largest eigenvalue the insertion of such parameters is not necessary, and we refer to [6] for the interpretation of these terms.

Let us introduce the function,

$$
U(x, s)=-\psi\left(x+\tau_{0}(\beta s), s\right),
$$

where $\beta=2^{(4 k-2) /(2 k+1)}$. Using the Bäcklund transformations we may write the largest eigenvalue distribution as,

$$
\begin{aligned}
\frac{d}{d s} \log \mathbb{P}^{(k)}(s) & =-\int_{-\infty}^{0} d x\left(\mathcal{L}_{k}\left[u_{1}\right]-\frac{1}{2} x\right)=\int_{-\infty}^{0} d x U\left(x-\tau_{0}(\beta s), s\right)^{2} \\
& =\int_{-\infty}^{-\tau_{0}(\beta s)} d x U(x, s)^{2}
\end{aligned}
$$

and we have that $U^{\prime}(x, s) / U(x, s)=W\left(x+\tau_{0}(\beta s), s\right)$ with $W(x, s)$ satisfying (3.42). Letting $q(x, s)=-W\left(x+\tau_{0}(\beta s), s\right)$ as well as using the known symmetry of the Painlevé equations (see e.g. [16]) we obtain the equations appearing in Theorem 1.12 of [12] in $q$ with $\alpha=+\frac{1}{2}$ and we have the relation,

$$
U^{\prime \prime}(x, s) / U(x, s)=q^{\prime}(x, s)+q^{2}(x, s) .
$$

This exactly reproduces the results of [12]. Note that we make the identification of our $\tau_{0}(s)$ with the function $-x(s)$ appearing in [12].

\section{Conclusions and Outlook}

In this paper we have presented an alternate derivation of the higher order analogues of the Tracy-Widom distribution which are characterised by the spectral density vanishing as a $(2 k-1) / 2$-root. Instead of using Fredholm determinants we apply orthogonal polynomials to directly calculate a truncated partition function for the matrix model in which the upper limit of the integration over the eigenvalues is finite. The gap probability for the matrix model can then be expressed in terms of this truncated partition function. This work therefore directly extends an earlier paper [14] in which the usual Tracy-Widom distribution was derived. Our results should also be compared with those obtained earlier in [12] via Riemann-Hilbert methods. We have shown the results presented here are equivalent to those appearing in [12] for odd values of $k$ via a sequence of Bäcklund transformations. One pleasing aspect of our approach is that the resulting higher order analogues of Tracy-Widom can be stated more succinctly than in [12]. Indeed we have,

$$
\log \mathbb{P}^{(k)}(s)=-\int_{-\infty}^{0} d x \frac{x}{2}\left(u_{1}(x, s)-u_{1}(x, \infty)\right)
$$

where $\mathbb{P}^{(k)}(s)$ is the gap probability at the $k$ th multicritical point for $k \in \mathbb{N}$, and $u_{1}(x, s)$ satisfies,

$$
\mathcal{L}_{k+1}^{\prime}\left[u_{1}\right]-4 s \mathcal{L}_{k}^{\prime}\left[u_{1}\right]=x u_{1}^{\prime}+2 u_{1}-2 s
$$


where $\mathcal{L}_{l}$ is the Lenard differential operator, see e.g. [6]. Here all derivatives denoted by prime are with respect to $x$. The issue of what boundary conditions to impose on $u_{1}$ are discussed in the main text but are only given implicitly in terms of the boundary conditions chosen in [12].

Let us emphasize that for technical reasons we had to restrict ourselves to a spectral density with one-arc support. However, we conjecture that the same generalised TracyWidom distributions will appear at suitably tuned inner or outer edges of a multi-arc support.

Finally, one advantage of avoiding the use of Fredholm determinants is that one should be able to calculate the probability distribution for large-deviations of the maximum eigenvalue from its mean. Such large deviations were the main focus of [14 and since our method is a direct extension of theirs we expect that such an analyse could be performed for the higher order cases. With our approach one could also investigate issues of universality in the large deviation tails. This would also give an alternative to the method in [19] which appears to also be able to address these questions. This is something we hope to pursue in future work.

Note: While completing this work an interesting paper appeared [20] in which partial differential equations for Fredholm determinants associated to the d.s.l of one and two matrix models were constructed using string equations and the method of pseudo-differential operators. Since the gap probability may be expressed as a Fredholm determinant there is a large overlap in the results presented here with those appearing in [20. However there are some important differences; firstly we give an explicit expression for the gap probability in terms of a solution to Painlevé XXXIV; hence our expression is in some sense a solution to the PDEs derived in [20]. However, on this point, it is not immediately clear how the PDEs appearing in [20] compare to our expressions as they seem to require the introduction of extra coupling constants in the potential which also scale non-trivially in the d.s.l. Finally, the Bäcklund transformation appearing here seems to partially answer the question raised in [20] concerning the relation between their approach and that of [12].

Acknowledgments: We acknowledge partial support by the SFB | TR12 "Symmetries and Universality in Mesoscopic Systems" of the German research council DFG (G.A.).

\section{A Equivalence of Gaussian Flow Equations}

The aim of this section is to prove the equivalence between our set of flow equations (2.25) and (2.26) for the Gaussian potential and a respective set of recurrence relations that was derived in ref. [14] in eqs. (42) and (41) there:

$$
\begin{aligned}
s_{n}-s_{n-1} & =-\frac{1}{2 \hat{\alpha}} \partial_{y} \log \left[r_{n}\right], \\
r_{n+1}-r_{n-1}+s_{n}^{2}-s_{n-1}^{2} & =-\partial_{\hat{\alpha}} \log \left[r_{n}\right],
\end{aligned}
$$

with $\hat{\alpha}=N \alpha$. Obviously the first equation agrees with (2.25). To show that eq. (A.2) also follows from our formalism consider first the matrix $P$ in the form of eq. (2.19). Due to its 
antisymmetry the diagonal has to vanish,

$$
\begin{aligned}
P_{n n} & =A_{n n}+C_{n n}-\hat{\alpha} B_{n n}=-\frac{1}{2} \partial_{y} \log \left[h_{n}\right]-\hat{\alpha} s_{n}=0 \\
\Rightarrow s_{n} & =-\frac{1}{2 \hat{\alpha}} \mathrm{e}^{-\hat{\alpha} y^{2}} \pi_{n}(y)^{2},
\end{aligned}
$$

where we have used (2.12) and (2.17) or equivalently (2.21) in the second line. Our main relation is obtained from the lower diagonal,

$$
\begin{aligned}
P_{n, n-1} & =A_{n, n-1}+C_{n, n-1}-\hat{\alpha} B_{n, n-1}=\frac{n}{\sqrt{r_{n}}}-\mathrm{e}^{-\hat{\alpha} y^{2}} \pi_{n}(y) \pi_{n-1}(y)-\hat{\alpha} \sqrt{r_{n}} \\
& =-P_{n-1, n}=\hat{\alpha} \sqrt{r_{n}} \\
\Leftrightarrow n & =\sqrt{r_{n}} \mathrm{e}^{-\hat{\alpha} y^{2}} \pi_{n}(y) \pi_{n-1}(y)+2 \hat{\alpha} r_{n},
\end{aligned}
$$

using eqs. (2.13) and (2.21) as well as the antisymmetry of $P$. We can now use the three step recurrence relation to eliminate $\sqrt{r_{n}} \pi_{n-1}(y)$ as well as use (A.3):

$$
\begin{aligned}
n & =\mathrm{e}^{-\hat{\alpha} y^{2}} \pi_{n}(y)\left(\left(y-s_{n}\right) \pi_{n}(y)-\sqrt{r_{n+1}} \pi_{n+1}(y)\right)+2 \hat{\alpha} r_{n} \\
& =-2 \hat{\alpha}\left(y-s_{n}\right) s_{n}-\left(n+1-2 \hat{\alpha} r_{n+1}\right)+2 \hat{\alpha} r_{n}, \\
\Leftrightarrow 2 n+1 & =2 \hat{\alpha}\left(\left(s_{n}-y\right) s_{n}+r_{n+1}+r_{n}\right),
\end{aligned}
$$

where we have also applied (A.4) for $n \rightarrow n+1$. Taking the difference between eq. (A.5) at $n$ and $n-1$ we finally arrive at

$$
\begin{aligned}
2 & =2 \hat{\alpha}\left(y\left(s_{n-1}-s_{n}\right)+s_{n}^{2}-s_{n-1}^{2}+r_{n+1}-r_{n-1}\right) \\
& =2 \hat{\alpha}\left(y \frac{1}{2 \hat{\alpha}} \partial_{y} \log \left[r_{n}\right]+s_{n}^{2}-s_{n-1}^{2}+r_{n+1}-r_{n-1}\right),
\end{aligned}
$$

upon inserting eq. (A.1). The last step to arrive at eq. (A.2) requires the following identity:

$$
1-\frac{y}{2} \partial_{y} \log r_{n}=-\hat{\alpha} \partial_{\hat{\alpha}} \log \left[r_{n}\right] .
$$

It follows by comparing the $y$ - and $\hat{\alpha}$ - derivative of

$$
1=\int_{-\infty}^{y} d \lambda \mathrm{e}^{-\hat{\alpha} \lambda^{2}} \pi_{n}(\lambda)^{2}=\frac{1}{\sqrt{\hat{\alpha}}} \int_{-\infty}^{\sqrt{\hat{\alpha}} y} d z \mathrm{e}^{-z^{2}}\left(\frac{1}{\sqrt{h_{n}}}\left(\frac{z}{\sqrt{\hat{\alpha}}}\right)^{n}+O\left(z^{n-1}\right)\right)^{2} .
$$

For the former we have already from (A.3)

$$
\partial_{y} \log \left[h_{n}\right]=\mathrm{e}^{-\hat{\alpha} y^{2}} \pi_{n}(y)^{2},
$$

whereas for the latter we obtain

$$
0=-\frac{1}{2 \hat{\alpha}}+\frac{y}{2 \hat{\alpha}} \mathrm{e}^{-\hat{\alpha} y^{2}} \pi_{n}(y)^{2}-\partial_{\hat{\alpha}} \log \left[h_{n}\right]-2 \frac{n}{2 \hat{\alpha}} .
$$

Inserting these equations into each other and taking the difference $\log \left[h_{n}\right]-\log \left[h_{n-1}\right]=\log \left[r_{n}\right]$ we arrive at (A.7). 


\section{B Multiplying Upper and Lower Triangular Matrices}

Any matrix $D$ can be decomposed into its strictly upper $(+)$, diagonal $(\mathrm{d})$, and strictly lower triangular (-) part:

$$
D=D_{+}+D_{\mathrm{d}}+D_{-}
$$

The aim of this appendix is to express the + and - part of the product of two matrices in terms of the,$+ \mathrm{d}$ and - parts of the individual factors, in order to express matrix $H$ in terms of matrix $P$ in the main text eq. (2.35). Obviously multiplying any matrix by the diagonal part of a matrix $D_{\mathrm{d}}$ will not change the character of that matrix to be,$+ \mathrm{d}$, or - :

$$
\left(B D_{\mathrm{d}}\right)_{+}=B_{+} D_{\mathrm{d}}, \quad\left(B D_{\mathrm{d}}\right)_{\mathrm{d}}=B_{\mathrm{d}} D_{\mathrm{d}}, \quad\left(B D_{\mathrm{d}}\right)_{-}=B_{-} D_{\mathrm{d}} .
$$

Furthermore multiplying two strictly upper (lower) triangular matrices gives a strictly upper (lower) triangular matrix, $\left(B_{+} D_{+}\right)_{+}=B_{+} D_{+}$and $\left(B_{-} D_{-}\right)_{-}=B_{-} D_{-}$.

¿From now on we will use that our matrix $B$ eq. (2.10) is tridiagonal. Therefore it can only shift off-diagonals at most up or down by one, that is

$$
\left(B D_{+}\right)_{+}=B_{+} D_{+}+B_{\mathrm{d}} D_{+}+\left(B_{-} D_{+}\right)_{+}=B D_{+}-\left(B_{-} D_{+}\right)_{\mathrm{d}},
$$

due to $\left(B D_{+}\right)_{\mathrm{d}}=\left(B_{-} D_{+}\right)_{\mathrm{d}}$ and $\left(B_{-} D_{+}\right)_{-}=0$. Analogously one can deduce

$$
\left(B D_{-}\right)_{-}=\left(B_{+} D_{-}\right)_{-}+B_{\mathrm{d}} D_{-}+B_{-} D_{-}=B D_{-}-\left(B_{+} D_{-}\right)_{\mathrm{d}},
$$

because of $\left(B D_{-}\right)_{\mathrm{d}}=\left(B_{+} D_{-}\right)_{\mathrm{d}}$ and $\left(B_{+} D_{-}\right)_{+}=0$. Consequently we can write down for any product $B D$ its respective,$+ \mathrm{d}$ and - part:

$$
\begin{aligned}
& (B D)_{+}=B D_{+}-\left(B_{-} D_{+}\right)_{\mathrm{d}}+B_{+} D_{\mathrm{d}}, \\
& (B D)_{\mathrm{d}}=\left(B_{+} D_{-}\right)_{\mathrm{d}}+B_{\mathrm{d}} D_{\mathrm{d}}+\left(B_{-} D_{+}\right)_{\mathrm{d}} \\
& (B D)_{-}=B D_{-}-\left(B_{+} D_{-}\right)_{\mathrm{d}}+B_{-} D_{\mathrm{d}} .
\end{aligned}
$$

We can now proceed expressing $H$ through $P$. Defining

$$
F=-\frac{N \alpha}{2} V^{\prime}(B)
$$

which commutes with $B$ and is symmetric as is $B$, we have

$$
P=F_{+}-F_{-},
$$

which has no diagonal part, $P_{\mathrm{d}}=0$. Finally we obtain

$$
\begin{aligned}
H & =(B F)_{+}-(B F)_{-}-y\left(F_{+}-F_{-}\right) \\
& =(B-y)\left(F_{+}-F_{-}\right)-\left(B_{-} F_{+}\right)_{\mathrm{d}}+\left(B_{+} F_{-}\right)_{\mathrm{d}}+B_{+} F_{\mathrm{d}}-B_{-} F_{\mathrm{d}} \\
& =(B-y) P-(B P)_{\mathrm{d}}+\left(B_{+}-B_{-}\right) F_{\mathrm{d}},
\end{aligned}
$$

where in the last step we have added trivially vanishing terms $\left(B_{-} P_{-}\right)_{\mathrm{d}}=0=\left(B_{+} P_{+}\right)_{\mathrm{d}}$ and $\left(B_{\mathrm{d}} P\right)_{\mathrm{d}}=0$ in order to express everything in terms of $P$. 


\section{The Double Scaling Limit in the Case $y \rightarrow \infty$}

Here we recall some standard results about the d.s.l of the Hermitian one-matrix model whose partition function is $Z_{N}\left(\infty ; \alpha,\left\{g_{l}\right\}\right)$. The equations (2.6) and (2.9) hold in this case with now all quantities independent of $y$. The recursion coefficients in the definition of (2.9) are determined by the recursion relations, also known as the string equations,

$$
\begin{aligned}
\alpha V^{\prime}(B)_{n, n+1} & =n / N, \\
V^{\prime}(B)_{n n} & =0 .
\end{aligned}
$$

Note that these equations can be obtained from (2.19) by setting derivatives of $y$, and hence $C$, to zero and using (2.13). By taking the large $N$ limit we can rewrite these equations in terms of the continuous variable $\xi=n / N$. It is known (see e.g. [7]) that for certain critical potentials in which the eigenvalue density vanishes as a $(2 k+1) / 2$-root at the right and a $\left(2 k^{\prime}+1\right) / 2$-root at the left end of the support, respectively, with $k^{\prime}<k$, that substituting the ansatz (3.1) and (3.2) into (C.1) results, after the rescaling (3.18), in the equations (3.4) and (3.5). It was then shown in [8] that the correct solution to (3.5) was $v_{1}=0$. The equations (C.1) may also be written as,

$$
[B, P]=1
$$

where $P$ is defined by (2.22). The equations (3.4) and (3.5) arise in this operator formalism since it is easy to show that $B$ scales to a differential operator independent of $k$,

$$
B \rightarrow B_{c}+a c_{1}\left(d^{2}+u_{1}(x)\right)+O\left(a^{2}\right)=B_{c}+a c_{1} \mathcal{B}+O\left(a^{2}\right) .
$$

Furthermore, due to the finite number of nonzero off-diagonals in $P$ and its antisymmetry, we expect $P$ to scale to an anti-hermitian differential operator. In general for $k^{\prime}<k$, $P \rightarrow a^{-\eta} \mathcal{P}+\ldots$ where $\eta>1$. Requiring that we have a scaling $\eta=1$ for the leading term determines the functions $v_{i}$, in particular it gives $(3.5)$. Given that $[\mathcal{B}, \mathcal{P}]$ must be a multiplicative operator we have $\mathcal{P} \propto\left(\mathcal{B}^{2 k-1}\right)_{+}$and therefore (3.4), up to rescalings.

\section{Derivation of the d.s.l for $H$}

In contrast to the main text in subsection 3.1 we first derive here the scaling of operator $P$, and then using the relation between $H$ and $P$ show the d.s.l for $H$ to be of the form (3.14). We now consider the case of the potential in $Z_{N}\left(y ; \alpha,\left\{g_{l}\right\}\right)$ taking the form of a critical potential of $Z_{N}\left(\infty ; \alpha,\left\{g_{l}\right\}\right)$. The left hand side of the flow equation (2.24) is exactly the left hand side of (‥2) and the right hand side of (2.24) has the same scaling dimension as the right hand side of (C.2). This means that using the ansatz (3.6) and (3.2), we have for $k^{\prime}<k$ that $v_{1}$ satisfies (3.5) and furthermore we can also conclude

$$
P \rightarrow a^{-1} \mathcal{P}+O(1)=a^{-1} c_{2}\left(\mathcal{B}^{(2 k-1) / 2}\right)_{+}+O(1) .
$$

The double scaling limit of the flow equation is then, using (3.15)

$$
1-\partial_{s} u_{1}=-4^{1-k} c_{1} c_{2} \mathcal{L}_{k}^{\prime}\left[u_{1}\right]
$$


which will be of use in the following. We now turn our attention to $H$ in (2.28) and prove,

$$
\mathcal{H}=c_{2} c_{1}\left(\left(\mathcal{B}^{(2 k+1) / 2}\right)_{+}-s\left(\mathcal{B}^{(2 k-1) / 2}\right)_{+}\right)+\frac{1}{4}\{x, d\}
$$

Recall the expression (2.35), we have,

$$
H=\frac{1}{2}\{B, P\}-y P-\frac{1}{2}\left\{B_{+}-B_{-}, \frac{N \alpha}{2}\left[V^{\prime}(B)\right]_{\mathrm{d}}\right\} .
$$

If we now consider the d.s.l it is a simple matter to prove that independent of $k$,

$$
B_{+}-B_{-} \rightarrow-2 c_{1} a^{1 / 2} d+O(a) .
$$

To find the double scaling limit of the operator $\frac{N \alpha}{2}\left[V^{\prime}(B)\right]_{\mathrm{d}}$ we must work slightly harder. Recalling the expression for $P(2.19)$ and (2.17), then the antisymmetry of $P$ implies,

$$
C_{n n}=-\partial_{y} \log \left[\sqrt{h_{n}}\right]=\frac{N \alpha}{2} V^{\prime}(B)_{n n}
$$

which gives,

$$
-\frac{1}{2} \partial_{y} \log \left[r_{n}\right]=\frac{N \alpha}{2} V^{\prime}(B)_{n n}-\frac{N \alpha}{2} V^{\prime}(B)_{n-1, n-1}
$$

where $V^{\prime}(B)_{n n}$ are trivially related to the operator $\left[V^{\prime}(B)\right]_{\mathrm{d}}$. In the large $N$ limit we have $\frac{N \alpha}{2} V^{\prime}(B)_{n n} \rightarrow \hat{V}(\xi, y)$, where $\hat{V}(\xi, y)$ is a continuous function of $\xi$. We therefore have,

$$
-\frac{1}{2} \partial_{y} \log [r(\xi, y)]=\frac{1}{N} \partial_{\xi} \hat{V}(\xi, y)
$$

Substituting in (3.6) and (3.2) while assuming an arbitrary scaling dimension for $\hat{V}$ i.e. $\hat{V}(\xi, y) \rightarrow a^{\eta} \mathcal{V}(x, s)$ gives,

$$
\frac{1}{4 c_{1}} \partial_{s}\left(u_{1}(x, s)+v_{1}(x, s)\right)=\frac{1}{4 c_{1}} \partial_{s} u_{1}(x, s)=a^{\eta+1 / 2} \partial_{x} \mathcal{V}(x, s),
$$

from which we see see that $\eta=-1 / 2$. Furthermore, using (D.2) we have,

$$
\mathcal{V}(x, s)=\frac{1}{4 c_{1}}\left(x+4^{1-k} c_{1} c_{2} \mathcal{L}_{k}\left[u_{1}\right]\right)
$$

where we have integrated and assumed the integration constant is zero.

Returning now to (D.4), the form of the d.s.l for all operators is now known. Substituting them in we obtain,

$$
\mathcal{H}=\frac{1}{2} c_{1} c_{2}\left\{\mathcal{B},\left(\mathcal{B}^{k-1 / 2}\right)_{+}\right\}-c_{1} c_{2} s\left(\mathcal{B}^{(2 k-1) / 2}\right)_{+}+\frac{1}{4}\{x, d\}+4^{-k} c_{1} c_{2}\left\{\mathcal{L}_{k}, d\right\}
$$

which upon using the identity [6] $\left(\mathcal{B}^{k+1 / 2}\right)_{+}=\frac{1}{2}\left\{\mathcal{B},\left(\mathcal{B}^{k-1 / 2}\right)_{+}\right\}+4^{-k}\left\{\mathcal{L}_{k}, d\right\}$ becomes (D.3). 


\section{E Shift Identity for Lenard Differential Operators}

We claim that when $z$ is a constant it holds for any integer $k \in \mathbb{N}$ :

$$
\mathcal{L}_{k}[u(x)+z]=\sum_{j=0}^{k}(4 z)^{k-j} \frac{\Gamma(k+1 / 2)}{\Gamma(k-j+1) \Gamma(j+1 / 2)} \mathcal{L}_{j}[u(x)] .
$$

We now prove this via induction. Firstly, it is trivially true for $k=1$. Now let us assume,

$$
\mathcal{L}_{k}[u(x)+z]=\sum_{j=0}^{k}(4 z)^{k-j} \alpha_{j}^{(k)} \mathcal{L}_{j}[u(x)],
$$

where we have defined

$$
\alpha_{j}^{(k)} \equiv \frac{\Gamma(k+1 / 2)}{\Gamma(k-j+1) \Gamma(j+1 / 2)}, \text { for } j=0,1, \ldots, k,
$$

and for any $k \in \mathbb{N}$. It is simple to see that the following identity holds for these constants:

$$
\alpha_{j}^{(k+1)} \equiv \alpha_{j}^{(k)}+\alpha_{j-1}^{(k)}, \text { for } j=1, \ldots, k .
$$

Let us now substitute the assumption (E.2) at $k+1$ into Lenard's recursion relation (3.16)

$$
\begin{aligned}
\mathcal{L}_{k+1}^{\prime}[u+z] & =\sum_{j=0}^{k}\left[\alpha_{j}^{(k)}(4 z)^{k-j} \mathcal{L}_{j+1}^{\prime}[u]+(4 z)^{k+1-j} \alpha_{j}^{(k)} \mathcal{L}_{j}^{\prime}[u]\right] \\
& =\sum_{j=1}^{k+1} \alpha_{j}^{(k+1)}(4 z)^{k+1-j} \mathcal{L}_{j}^{\prime}[u] \\
\Rightarrow \mathcal{L}_{k+1}[u+z] & =\sum_{j=1}^{k+1} \alpha_{j}^{(k+1)}(4 z)^{k+1-j} \mathcal{L}_{j}[u]+C_{k+1}
\end{aligned}
$$

Here we have introduced $C_{k+1}$ as an integration constant and used the fact that $\mathcal{L}_{0}$ is a

constant as well as that $\alpha_{k+1}^{(k+1)}=\alpha_{k}^{(k)}=1$. To complete the proof by induction we must fix $C_{k+1}$. This can be done by using the fact that $\mathcal{L}_{j}[0]=0$ for all $j>0$, so that we have,

$$
\mathcal{L}_{k+1}[z]=C_{k+1} .
$$

Due to the homogenous scaling of $\mathcal{L}_{k+1}[z]$ we know there exists only one term in $\mathcal{L}_{k+1}[z]$ which is non-zero when $u(x)=z=$ const; it is, $\beta_{k+1} u^{k+1}$, where $\beta_{k+1}$ is a known constant that follows from the properties of the Lenard differential. We therefore have,

$$
C_{k+1}=\beta_{k+1} z^{k+1} .
$$

Let us finally determine $\beta_{k}$. This can be done by noting that in the Lenard recursion relation, the only term in $\mathcal{L}_{k}$ which contributes to the $u^{k+1}$ term in $\mathcal{L}_{k+1}$, is $\beta_{k} u^{k}$. Hence we have from the Lenard recursion relation,

$$
\begin{aligned}
d\left(\beta_{k+1} u^{k+1}\right) & =4 u \beta_{k} u^{k-1} u^{\prime}+2 u^{\prime} \beta_{k} u^{k}=4 \frac{k+\frac{1}{2}}{k+1} \beta_{k} d\left(u^{k+1}\right) \\
\Rightarrow \beta_{k+1} & =4^{k} \frac{\Gamma(k+3 / 2)}{\Gamma(k+2) \Gamma(3 / 2)} \beta_{1}
\end{aligned}
$$


where $\beta_{1}=1$. Substituting the known value of $C_{k+1}$ into our previous relations using $\mathcal{L}_{0}=1 / 2$ gives,

$$
\mathcal{L}_{k+1}[u+z]=\sum_{j=0}^{k+1} \alpha_{j}^{(k+1)}(4 z)^{k+1-j} \mathcal{L}_{j}[u]
$$

thereby completing the proof.

\section{References}

[1] C. A. Tracy and H. Widom, Proceedings of the ICM, Beijing 2002, vol. 1, 587-596 [arXiv:math-ph/0210034v2].

[2] C. A. Tracy and H. Widom, Phys. Lett. B305 (1993) 115-118; Commun. Math. Phys. 159 (1994) 151-174.

[3] P. J. Forrester, Nucl. Phys. B402 [FS] (1993) 709-728.

[4] P. Deift, T. Kriecherbauer, K. T.-R. McLaughlin, S. Venakides, and X. Zhou, Comm. Pure Appl. Math. 52 (1999) 1335-1425.

[5] A. Soshnikov, Comm. Math. Phys. 207 (1999) 697-733.

[6] P. Di Francesco, P. Ginsparg, and J. Zinn-Justin, Phys. Rept. 254 (1995) 1-133 [arXiv:hep-th/9306153v2].

[7] G. W. Moore, Prog. Theor. Phys. Suppl. 102 (1990) 255-285.

[8] S. Dalley, C.V Johnson, and T. Morris, Mod. Phys. Lett. A6 (1991) 439-448.

[9] T. Claeys and M. Vanlessen, Comm. Math. Phys. 273 (2007) 499-532 [arXiv:mathph/0607043v1].

[10] M. Adler, M. Cafasso, and P. van Moerbeke, Nonlinear PDEs for gap probabilities in random matrices and KP theory, Physica D: Nonlinear Phenomena, August 2012 in press, [arXiv:1104.4268v2 [math-ph]].

[11] M. Bergère and B. Eynard, Universal scaling limits of matrix models, and (p,q) Liouville gravity, arXiv:0909.0854v1 [math-ph].

[12] T. Claeys, A. Its, and I. Krasovsky, Comm. Pure Appl. Math. 63 (2010) 362-412 [arXiv:0901.2473v1 [math-ph]].

[13] T. Claeys and S. Olver, Contemporary Mathematics, J. Arves and G. Lpez Lagomasino (eds.), Recent Advances in Orthogonal Polynomials, Special Functions, and Their Applications, 83-99 [arXiv:1111.3527v1 [math-ph]]. 
[14] C. Nadal and S. N. Majumdar, J. Stat. Mech. (2011) P04001 [arXiv:1102.0738v3 [condmat.stat-mech]].

[15] A. Das, "Integrable Models", World Scientific, Lecture Notes in Physics Vol. 30, Singapore, 1989.

[16] P. A. Clarkson, N. Joshi, and A. Pickering, Inverse Problems 15 (1999) 175-188 [arXiv:solv-int/9811014v1].

[17] M. L. Mehta, "Random Matrices", Academic Press, Third Edition, London 2004.

[18] M. Douglas and S. Shenker, Nucl. Phys. B335 (1990) 635-654; E. Brézin and V. Kazakov, Phys. Lett. B236 (1990) 144-150; D. Gross and A. Migdal, Phys. Rev. Lett. 64 (1990) 127-130; Nucl. Phys. B340 (1990) 333-365.

[19] G. Borot, B. Eynard, S. N. Majumdar, and C. Nadal, J. Stat. Mech. (2011) P11024 [arXiv:1009.1945v4 [math-ph]]; G. Borot and C. Nadal, Right tail expansion of TracyWidom beta laws, arXiv:1111.2761v1 [math-ph].

[20] M. Adler, M. Cafasso, and P. van Moerbeke, Nonlinear PDEs for Fredholm determinants arising from string equations, arXiv:1207.6341v1 [math-ph]. 